\title{
Seismic risk assessment of the Middle Amur lowland
}

\author{
Viktor Shabalin ${ }^{1, *}$, Aleksandr Zhuravlev ${ }^{1}$ Jaroslav Sheetz ${ }^{1}$, and Stanislav Gilmutdinov ${ }^{1}$ \\ ${ }^{1}$ Far Eastern State Transport University, 47 Seryshev St,.Khabarovsk, 680021, Russia
}

\begin{abstract}
This article presents the results of seismic process studies in the territories adjacent to the essential facilities of the Far East. It shows the possibility for a reasonable description of the epicenter vector during prediction seismic impacts on extended objects.
\end{abstract}

\section{Introduction}

Rational ground of engineering solutions in seismic areas requires a quantitative description of seismicity. This description should correspond to the ways of its continued use: in some cases it is necessary to provide simultaneous prediction for expected intensity in different places, in other cases - independent evaluations of possible earthquake impacts in each considered area will be sufficient.

Final goal of these engineering solutions is earthquake-resistant construction. According to the Set of Rules 14.13330.2014 [5] in construction design for seismic areas the materials and structural design providing the lowest value of earthquake loads have to be applied. It must lead to significant rise in a projects price. Works on seismic microzonation made by instrumental methods which goal is to divide an area into parts with different expected intensity of reaction to seismic impact should precede any design. The above-mentioned reaction is set in terms of intensity on a scale MSK-64 as well as peak seismic accelerations, velocities and movements for residual deformation prediction. However, besides nearsurface geological conditions it is necessary to mention that peak ground reactions and constructions are influenced by nature of an oncoming seismic signal as well. Such parameters as vector of seismic waves, amount of seismic energy released at depth, its scattering on crustal heterogeneity influence directly on frequency, amplitude and duration of seismic effect. Their prediction along with the results of seismic microzonation is the basis of engineering seismological prediction. Thereby, besides experimental researches directly on the object it is necessary to study characteristics of seismic process of the territories adjoined to an object and region in general.

\section{Initial data and processing methods}

Materials about earthquakes in a zone of the Baikal-Amur Mainline [4] as well as any other region can be presented in three groups:

\footnotetext{
*Corresponding author: Viktor.Shabalin@gmail.com
} 
1. Reports on strong earthquakes from written sources, paleo-seismological reconstructions [1];

2. Instrumental data on earthquakes obtained under conditions of changing observation network - unsteady recording procedures, nonstandard processing procedures of seismograms, etc.;

3. Instrumental information gained with the help of modern methods during a long period of time which are comparable in level of errors in determining main parameters.

The initial data were received from three sources: Massachusetts Institute of Technology (USA), Hokkaido Institute of Seismology (Japan), Institute of Tectonics and Geophysics FEB RAS (Russia). The investigated area is limited $\lambda[300: 750], \varphi[1170: 1800]$.

\section{Recurrence of Earthquake}

One of the most important characteristics of seismic mode is a recurrence chart, where function $N(E)$ with fixed value $\Delta t$; here $N$ is a number of earthquakes. Dependence $N(E)$ in logarithmic scale is initially lineal. It's approximated by the following ratio:

$$
\lg \mathrm{N}=\gamma \mathrm{K}+\mathrm{A}
$$

Here A is a numerical parameter characterizing a level of earthquakes;

$\gamma$ - tilt of a recurrence chart (seismicity rate) that characterize seismic activity in a region. In figure 1 there is a recurrence chart of the considered region since 1970 to the present.

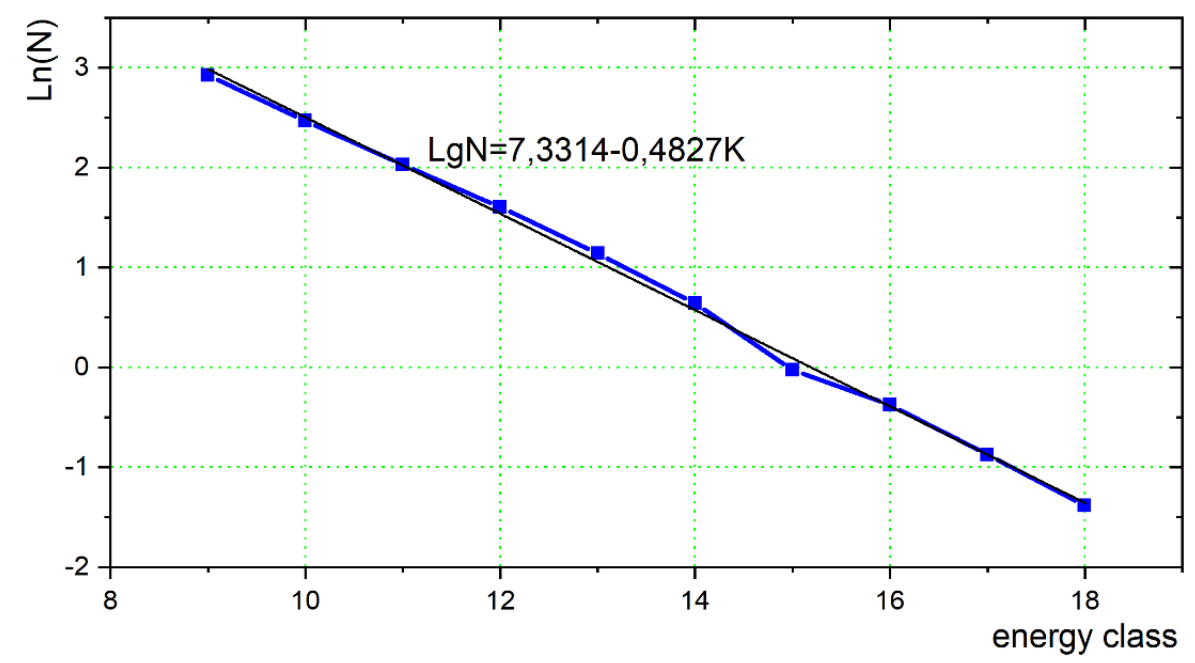

Fig. 1. Chart of recurrence for the region of the Middle Amur lowland.

For the evaluation of a long-term middle recurrence they adhere to the assumption about the same middle level of seismic activity in the region and extrapolate earthquake data to the whole zone.

\section{Assessment of Seismic Activity A10}

Value of A10 can be rated by two ways [3]:

1. According to constant comprehensiveness sizes of averaging areas are accepted as identical throughout the territory. The area is covered by a rectangular even grid (e.g. in $0,4-0,5$ degree latitude and longitude) and activity value is determined in its nodes. The 
averaging areas with the centers in nodes can be arranged very close or overlapping in all directions (moving averaging). A number of epicenters in these areas can be taken from epicenter maps or earthquake catalogues.

2. According to constant accuracy sizes of averaging areas are chosen so that each area contains the same fixed number of epicenters. Practically these areas are made circular.

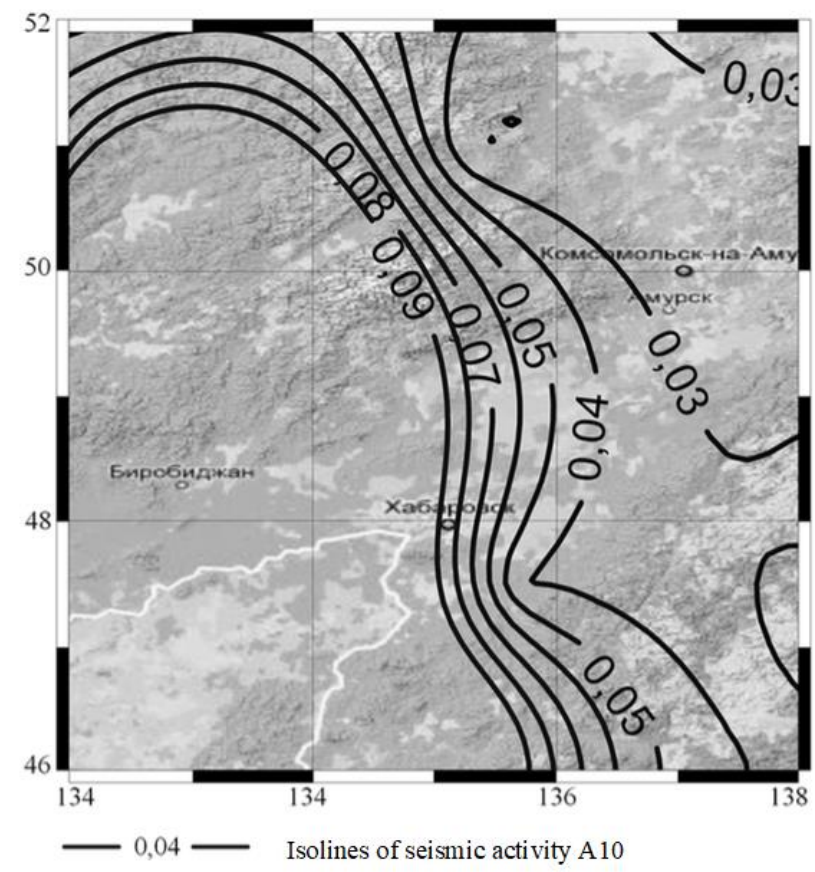

Fig. 2. A part of a seismic activity map of Middle Amur lowland.

The map of seismic activity A10 for the sampled territory is made by a summing method according to the formula of Riznichenko [2]:

$$
A_{10}=\frac{1-10^{-\gamma}}{10^{-\gamma}\left(\mathrm{K}_{\min }-\mathrm{K}_{10}\right)} \cdot \frac{\mathrm{S}_{0} \mathrm{~T}_{0}}{\mathrm{~S} \cdot \mathrm{T}} \cdot \mathrm{N}_{\Sigma}
$$

Where $\gamma$ - tilt of recurrence chart;

Kmin - level of representation class;

$\mathrm{K} 10$ - class to which corresponds calculated activity;

$\mathrm{S}$ - size of averaging area;

$\mathrm{S} 0$ - rate unit in terms of area accepted in accordance with A10;

$\mathrm{T}$ - earthquake observation period;

T0 - unit of time;

$\mathrm{N} \Sigma$ - total number of earthquakes of different energy classes $\mathrm{K} \geq \mathrm{Kmin}$, observed on area $\mathrm{S}$ during a period of time $\mathrm{T}$.

Physical meaning of the calculated value A10 can be represented as follows: A10 is a number of 10th energy class earthquakes which occur at a site of 1000 sq.km. It's a specification of an observation stage with the results indicated in the used earthquake catalogue.

The processing data includes earthquakes with minimum value of energy class 12 $(\mathrm{M}>4.4)$. A map for seismic activity A10 for the reviewed territory is made by a method of summing up. Physical meaning of the shown contour lines values A10 on the map can be formulated as follows: A10 is a number of 10th energy class earthquakes (M>3.3) which 
occur at a site of 1000 sq.km. It's a specification of an observation stage with the results indicated in the used earthquake catalogue.

The territory covers an area of the Middle Amur lowland. Generally it can be seen that the area under the consideration demonstrates less seismic activity than territories to the west and south-west of it, and some more seismic activity than areas to the east of it. And besides the activity tends to decrease from the south-west to the north-east from A10 $=0.09$ till $\mathrm{A} 10=0.03$. In this way for almost 50 years of seismic observations the area of the Middle Amur lowland has been demonstrating seismic activity from medium to low. Seismic potential has been realized in weak earthquakes with a complete absence of large events.

\section{Conclusion}

Summarizing the seismic analysis performed in the Middle Amur lowland of the Far East it is possible to draw a conclusion that there is an opportunity for a justified description of a seismic focus vector while predicting seismic impacts on essential facilities and estimation of seismic risk.

The study results made for the area of extended transport structures show an opportunity of a large magnitude seismic event $(M>5)$ to the south and to the west of it. Although it is possible that demonstration of a cluster seismicity can remove the tension level in a intersection node of seismogenic zones without bringing the situation to a large earthquake.

\section{Reference}

1. V.A. Bezgodkov, R.E. Chavshuyan, Earthquake prediction 10, 193-205 (1988)

2. S.V. Mishin, Seismic process and retention of pulse (NECSRI FEB RAS, Magadan, 2004)

3. E.F. Savarenskiy, D.P. Kirnos, Elements of seismology and seismometry (State publishing house for technical and theoretical literature, Moscow, 1945)

4. V.P. Solonenko, V.V. Nikolaev, R.M. Semenov, Geology and seismicity of the BAM zone. Seismology and seismic zonation (Science, Novosibirsk, 1985)

5. Russian State Standard SR 14.13330.2014 Construction in seismic areas 